\title{
Intracellular infection with Spironucleus barkhanus (Diplomonadida: Hexamitidae) in farmed Arctic char Salvelinus alpinus
}

\author{
Erik Sterud $^{1, *}$, Trygve Poppe ${ }^{2}$, Geir Bornø ${ }^{3}$ \\ ${ }^{1}$ National Veterinary Institute, PO Box 8156 Dep., 0033 Oslo, Norway \\ ${ }^{2}$ Norwegian School of Veterinary Science, PO Box 8146 Dep., 0033 Oslo, Norway \\ ${ }^{3}$ National Veterinary Institute, 9480 Harstad, Norway
}

\begin{abstract}
A case of intracellular systemic infection with the diplomonad flagellate Spironucleus barkhanus in farmed Arctic char Salvelinus alpinus is described. The parasites were widely disseminated throughout the vasculature and in most organs. Aggregates of the parasites were seen within well-defined structures regarded as host cells in capillaries and sinusoids of the liver, spleen and head kidney. Intracellular infection with Spironucleus spp. has never previously been reported. The prevalence of infection and mortality in the affected farm was low. In contrast to systemic spironucleosis in farmed Atlantic salmon, and despite huge numbers of flagellates in the vasculature, the tissues of the organs were remarkably unaffected. The relatively few gross and histopathological lesions may indicate that Arctic char are more tolerant to this parasite than Atlantic salmon.
\end{abstract}

KEY WORDS: Arctic char - Diplomonadida - Hexamitids - Pathology · Salvelinus alpinus . Spironucleus barkhanus

\section{INTRODUCTION}

The history of systemic spironucleosis in farmed salmonids is peculiar. The first cases were reported from Atlantic salmon Salmo salar (L.) in Norway from 1989 to 1991 (Mo et al. 1990, Poppe et al. 1992), and concurrently from Chinook salmon Oncorhynchus tshawytscha in British Columbia, Canada (Kent et al. 1992). First believed to be a Hexamita species, the flagellate was later described as Spironucleus barkhanus (Sterud et al. 1997). Sterud et al. (1998) hypothesised that Atlantic salmon were infected by flagellates transmitted from wild Arctic char Salvelinus alpinus. However, despite the fact that Spironucleus barkhanus is commonly found in wild char in fish-farming regions of Norway (authors' pers. obs.), no new cases of spironucleosis in farmed salmonids have been reported until now.

In October 2001, systemic infection with Spironucleus barkhanus was observed in market-sized $(3+\mathrm{kg})$
Atlantic salmon in a seawater fish farm near Alta in northern Norway. The overall pathology was as described by Poppe et al. (1992), and the outbreak culminated in the destruction of $640 \mathrm{t}$ of salmon. In March 2002, the National Veterinary Institute (NVI) in Oslo received histological material from farmed Arctic char from northern Norway. A massive systemic and intracellular infection with $S$. barkhanus was confirmed in these fish. This case, discussed here, represents the first documented case of systemic spironucleosis in farmed Arctic char and the first report of a Spironucleus species as an intracellular parasite.

\section{MATERIALS AND METHODS}

The affected fish farm is located in Vesterålen in northern Norway $\left(68^{\circ} 35^{\prime} \mathrm{N}, 15^{\circ} 30^{\prime} \mathrm{E}\right)$ and produces Arctic char Salvelinus alpinus (L.) in net cages in the sea. A small number of fish with aberrant swimming 
and exophthalmia were observed during a routine visit by the local fish health service in March 2002. The mortality was low and considered by the farmer to be unproblematic. Unidentified parasites were found in histological sections from these fish and the NVI was sent haematoxylin and eosin (H\&E) stained histological sections of 3 char hearts for identification of the parasites. A closer examination was found to be necessary, and 11 char (300 to $800 \mathrm{~g}$ ) including both fish with and without clinical signs of disease were sampled, killed by a blow to the head and shipped on ice to the NVI. During the autopsy, fresh smears and imprints of blood and tissues were viewed in a phase-contrast light microscope. Blood drawn from the caudal vein and tissue samples from internal organs were fixed in $10 \%$ neutral buffered formalin or $3 \%$ gluataraldehyde in 0.1 M cacodylate buffer ( $\mathrm{pH}$ 7.4). Samples fixed in formalin were routinely processed for histology, and $5 \mu \mathrm{m}$ paraffin sections were stained with H\&E. Samples fixed in glutaraldehyde were post-fixed in $1 \%$ osmium tetroxide, dehydrated in a graded ethanol series and prepared for scanning and transmission electron microscopy (SEM and TEM). Blood samples were dispersed on Nuclepore filters that were subsequently prepared for SEM through critical-point drying and gold-coating. Samples were viewed in a JEOL JSM 6400 scanning electron microscope. Tissue samples for TEM were embedded in Ladd Lx 100 resin, and ultrathin sections stained with uranyl acetate and lead citrate were viewed in a Philips EM 208S transmission electron microscope.

For bacteriology, samples from the anterior part of the kidney were streaked onto blood agar plates with and without additional supplementation of $2 \%$ $\mathrm{NaCl}$, and incubated at 15 and $22^{\circ} \mathrm{C}$ respectively for $6 \mathrm{~d}$.

Cultivation of the parasites was attempted. Blood (1 ml) drawn from the caudal vein and small pieces of liver and kidney were suspended in $15 \mathrm{ml}$ cultivation tubes containing $9 \mathrm{ml}$ Keister's medium (bilesupplemented TYI-S-33 medium; Keister 1983), incubated at $5^{\circ} \mathrm{C}$, and checked daily for the presence of live flagellates under a dissection microscope.

\section{RESULTS}

Large numbers of flagellates, similar to those seen in the H\&E-stained sections of the hearts sent to the NVI, were found in fresh smears of blood and organs of 5 of the 11 fish examined (Fig. 1). They were identified as hexamitid flagellates, being equipped with 2 anterior nuclei, and 6 anterior and 2 posterior flagella. They were oval to pyriform in shape and measured $8.5 \times$ $3.4 \mu \mathrm{m}(\mathrm{n}=10)$.

\section{Histopathology}

Heart. Densely packed hexamitids were visible between the bundles of fibro-elastic tissue in the wall of the bulbus arteriosus of infected fish (Fig. 2). No degenerative changes or cellular reaction associated with the parasites were found. Similar flagellates, but more scattered, were found together with erythrocytes in the ventricular lumen, in the coronary vasculature of the ventricle, and in the spongious part of the ventricular myocardium (Figs. 3 \& 4). In some areas we observed a mild to moderate cellular response, comprising lymphocytes and macrophages in the spongiosa, and a limited hypertrophy of the endocardial lining.

In most hearts, there was a marked thickening of the epicardium due to proliferation of connective tissue and infiltration of inflammatory cells. In some areas, the thickened epicardium showed characteristic nodular protrusions.

A marked formation of layered thrombi in the lumen of the bulbus arteriosus and in the atrium was observed.

Eye. The vascular plexus of the choroid gland was densely packed with hexamitid flagellates (Fig. 5) which, in some vessels, appeared to replace the erythrocytes. Larger arteries in the area were also filled with parasites.

Liver. The liver was severely congested and oedematous and extensive vacuolisation of the hepatocytes was observed. Numerous densely packed multicellular aggregates with a defined demarcation were diffusely scattered throughout vessels, in dilated sinusoids and between parenchymatic cells (Figs. 6 to 8). Each aggregate, comprising a single dark 'body' and a varying number of lighter stained cells with characteristic

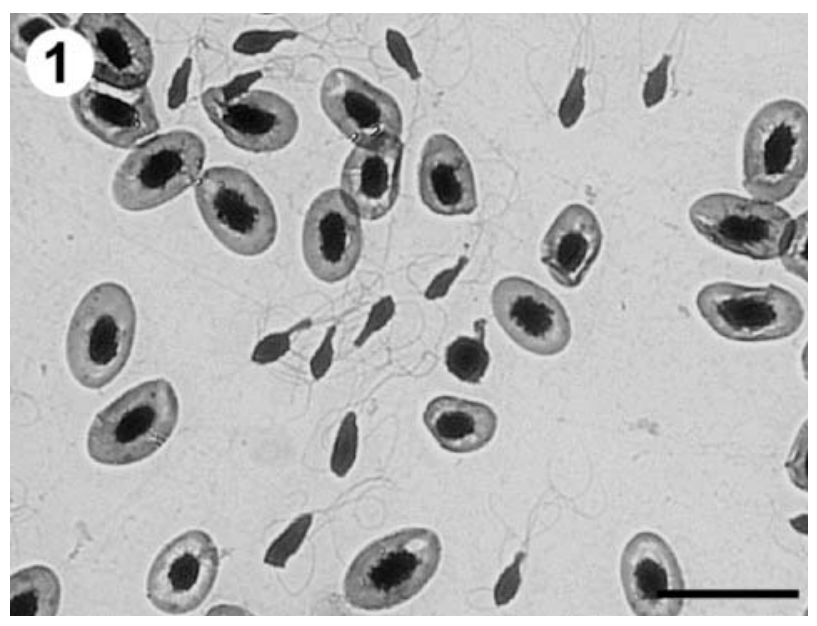

Fig. 1. Salvelinus alpinus. Air-dried and Diff Quick ${ }^{\circledR}$-stained blood smear from Arctic char infected with Spironucleus barkhanus. Scale bar $=10 \mu \mathrm{m}$ 

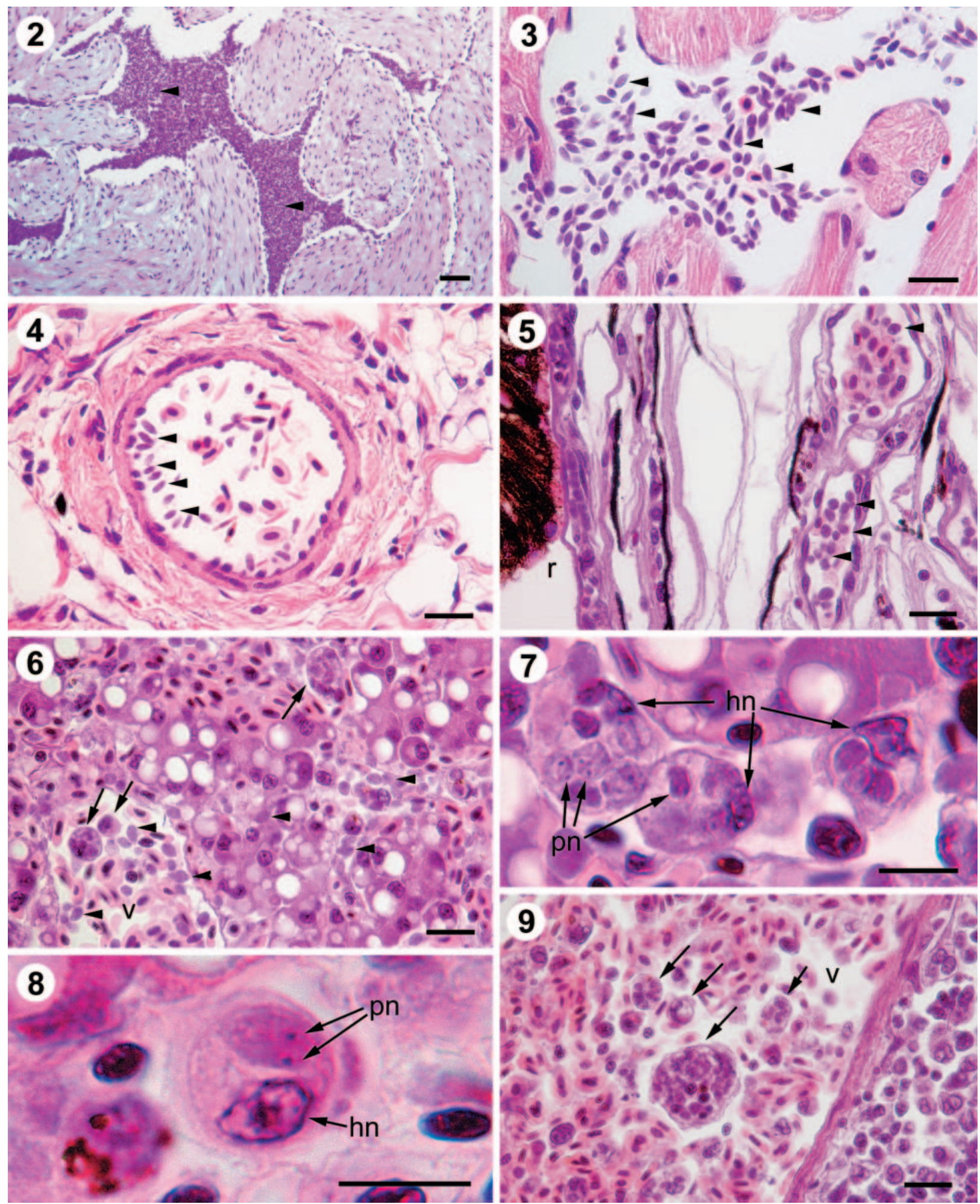

Figs. 2 to 9. Salvelinus alpinus. Light micrographs of histological sections from Arctic char infected with Spironucleus barkhanus stained with haematoxylin and eosin. Fig. 2. Numerous trophozoites of $S$. barkhanus (arrowheads) within lumen of bulbus arteriosus. Fig. 3. Trophozoites of $S$. barkhanus (arrowheads) within ventricular lumen. Fig. 4. Trophozoites of $S$. barkhanus (arrowheads) within lumen of coronary artery. Fig. 5. Trophozoites of $S$. barkhanus (arrowheads) within vessels of choroid plexus of the eye; $\mathrm{r}=$ retina. Fig. 6 . Trophozoites of $S$. barkhanus (arrowheads) and host cells (arrows) with intrecellular stages of $S$. barkhanus in vessels and sinusoids of the liver. Fig. 7. Three host cells in liver sinusoid with varying numbers of intracellular stages of $S$. barkhanus; hn = host cell nucleus, pn = flagellate nucleus. Fig. 8. Single $S$. barkhanus inside host cell; hn $=$ host cell nucleus, pn = flagellate nucleus. Fig. 9. Host cells (arrows) with varying numbers of intracellular stages of $S$. barkhanus, within lumen of a vein (v) in the head-kidney. Scale bars in (2-6) and (9) $20 \mu \mathrm{m}$; scale bars in (7 \& 8) $=10 \mu \mathrm{m}$ 
paired dark 'eyes', was subsequently identified as a host cell containing intracellular stages of hexamitid flagellates (Figs. $7 \&$ 8). Single trophozoites were also present in vast numbers in the same locations. (Fig. 6).

Kidney. Parasitised host cells similar to those described from the liver were diffusely scattered throughout dilated sinusoids and vessels of the head- and mid-kidney (Fig. 9). In the mid-kidney there were, in addition, large numbers of trophozoites throughout vessels and sinusoids. There was only a mild inflammatory response in the haematopoietic tissue in the head-kidney, while diffuse necrosis of the interstitial haematopoietic tissue was present adjacent to the sinusoids of the mid-kidney.

Spleen. The entire spleen was congested and diffusely infiltrated with free-swimming and intracellular hexamitids similar to those in the liver and kidney. Smaller vessels were almost obliterated by trophozoites while intracellular stages were present in vast numbers in the larger vessels. There was widespread necrosis of the splenic pulp where virtually no normal tissue could be identified.

Posterior gut. There was severe congestion and diffuse necrosis of the lamina propria and a mild to moderate inflammatory response of the stratum granulosum. Vessels were packed with hexamitids. No organisms could be identified in the intestinal lumen.

Other organs. Other organs examined included the pyloric caecae with pancreas, gills, pseudobranchs, brain and gonads. Blood vessels in these organs were packed with flagellates. With this exception, no pathological changes were seen in the tissues.

\section{Electron microscopy}

Scanning electron microscopy. Scanning electron microscopy of blood samples showed oval to pyriform hexamitid flagellates, measuring $8 \times 3.8 \mu \mathrm{m}(\mathrm{n}=10)$, and possessing 6 anterior and 2 posterior flagella, among the red blood cells (Fig. 10). Each posterior flagellum emerged through a cytostome, adorned by a low crescent-shaped ridge; these ridges were oriented in opposite directions in the 2 openings (Fig. 11). Other surface adornments were not seen.

Transmission electron microscopy. The flagellates viewed by TEM had 2 prominent nuclei at the anterior end. These were intertwined at their anterior ends, giving a characteristic s-shape in the longitudinal section (Fig. 12). The 2 recurrent flagella ran between the nuclei (Fig. 13) and were accompanied by a striated lamina and 3 bands of microtubules. In the crosssection these bands appeared as a spoke-like pattern (Fig. 14). The maximum number of microtubules in the 3 bands were $10+2+5$.
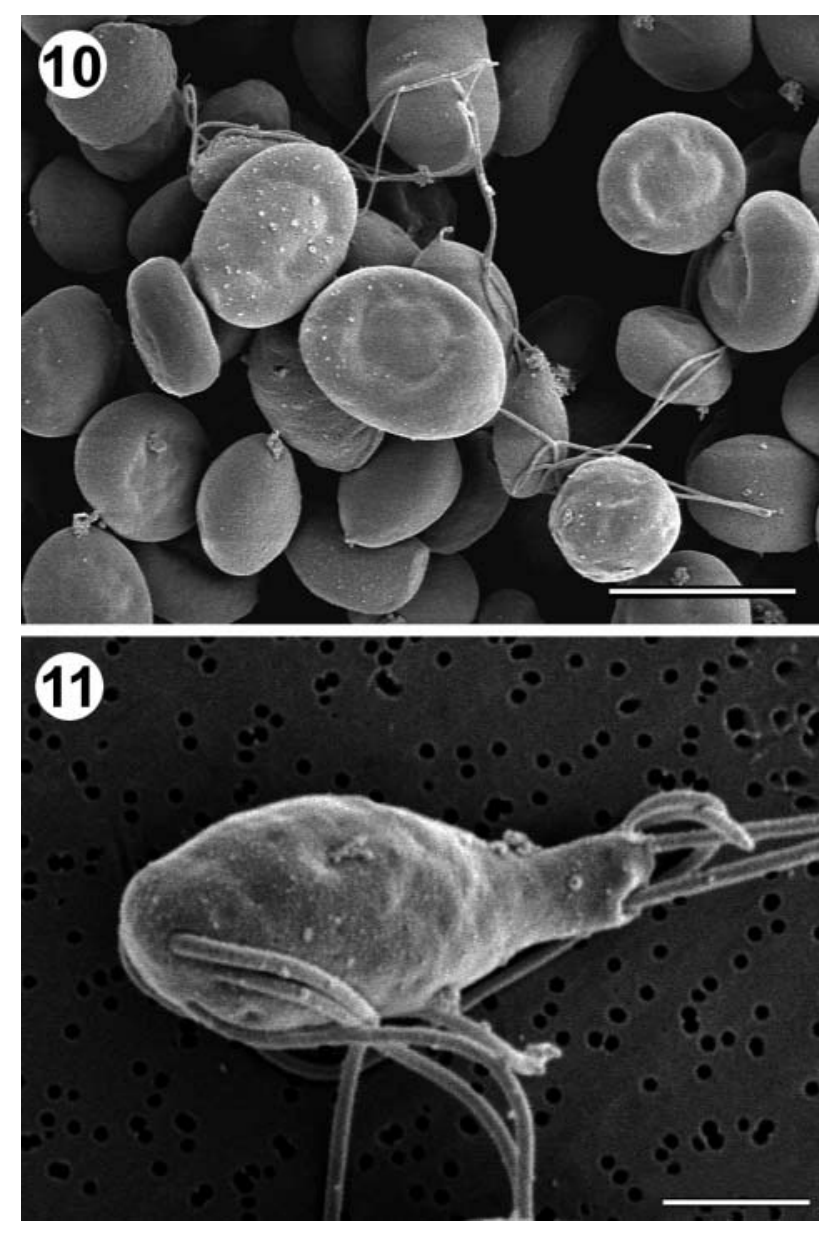

Figs. 10 \& 11. Salvelinus alpinus. Scanning electron micrographs of blood from Arctic char showing presence of trophozoites of Spironucleus barkhanus. Scale bar in $10=10 \mu \mathrm{m}$; in $11=2 \mu \mathrm{m}$

The intracellular stages visible under light microscopy were also visible under the TEM (Figs. 15 \& 16). These flagellates typically had their flagella wrapped around them, giving them a characteristic appearance in the cross-section (Figs. 16 \& 17). Some flagellates had 2 recurrent flagella in the cross-section (Fig. 16), while 4 were visible in others, indicating dividing cells. In 1 case a flagellate was observed inside an erythrocyte (Fig. 18).

\section{Microbiology and cultivation}

No bacteria were isolated from affected fish. Attempts to cultivate the flagellates from blood failed. After $4 \mathrm{~d}$ no live flagellates were visible in the culture tubes. 

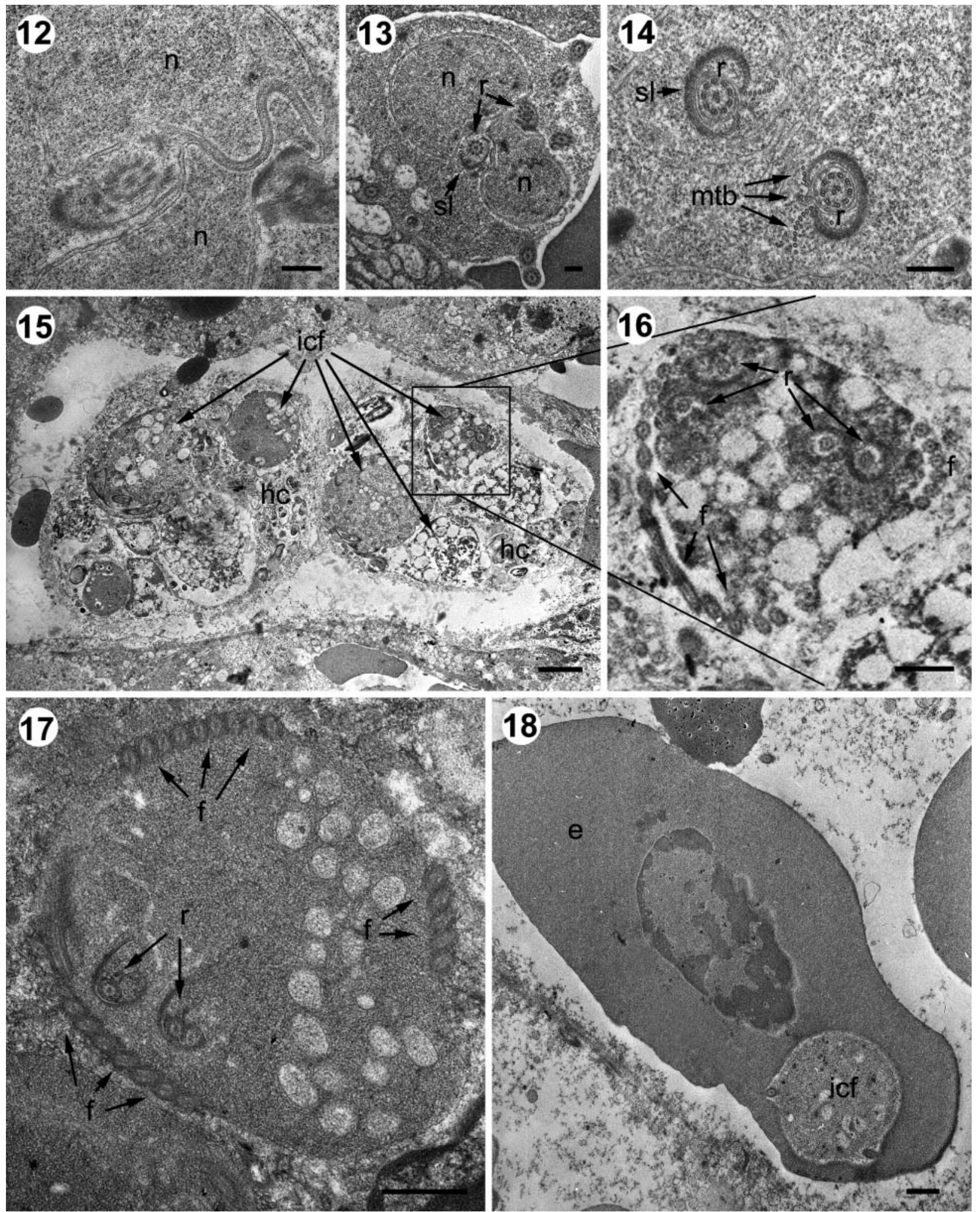

Figs. 12 to 18. Salvelinus alpinus. Transmission electron micrographs of Spironucleus barkhanus within liver and kidney tissue of Arctic char. Fig. 12. Oblique section through anterior end showing 2 nuclei (n) closely apposed. Fig. 13. Cross-section through anterior end showing 2 recurrent flagella $(\mathrm{r})$ passing between the 2 nuclei $(\mathrm{n})$; sl = striated lamina. Fig. 14. Cross-section of middle region showing arrangement of microtubular bands ( $\mathrm{mtb}$ ) and striated lamina (sl) accompanying the recurrent flagella (r). Fig. 15. Several intracellular flagellates (icf) within unidentified host cells (hc) in a liver capillary. Fig. 16. Close-up of 1 individual flagellate from Fig. 15; r = recurrent flagellum, $\mathrm{f}=$ flagella. Fig. 17. Cross-section of intracellular specimen; note characteristic

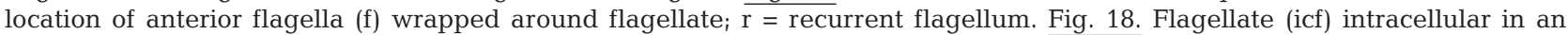

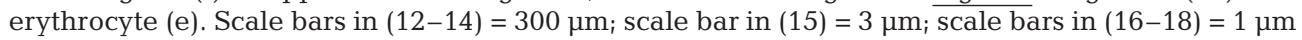




\section{DISCUSSION}

Based on the morphology and ultrastructure as viewed by SEM and TEM, the hexamitid flagellates found in Arctic char Salvelinus alpinus in the present study were identified as Spironucleus barkhanus. The crescent-shaped ridges (barkhans) around the cytostomes, and the cross-sectional pattern of the microtubular bands accompanying the recurrent flagella, have only been seen in this species (Sterud et al. 1997, 1998). $S$. barkhanus is commonly found in the gall bladder and intestine of wild Arctic char and grayling Thymallus thymallus, and is also known as the cause of severe systemic infection in farmed Atlantic salmon (Poppe et al. 1992, Sterud et al. 1997, 1998). Its presence as a pathogen in farmed Arctic char has never previously been documented. However, during the present study we became aware that the affected fish farm has experienced regular outbreaks of systemic spironucleosis over the last $10 \mathrm{yr}$ (E. Sommerhaug pers. comm.). According to the farmer, the disease has mainly affected smaller fish in poorer than average condition. The mortality in such cases has been modest and the outbreaks have faded, leaving the farmer with the impression of a fairly unproblematic disease. This is in contrast to systemic infection with $S$. barkhanus in Atlantic salmon. All known cases with farmed Atlantic salmon have been characterised by massive morbidity and mortality. The prevalence of infection has been very high, approaching $100 \%$ in some affected units. Systemic spironucleosis in Atlantic salmon is characterized by sub-cutaneous and muscular boil lesions with putrid contents as well as by extensive necrotic changes in liver, kidney and spleen (Poppe et al. 1992). Such lesions appeared to be absent from char, despite the massive presence of the parasite in the vasculature of most organs. The extensively vacuolated livers seen in affected fish are considered normal in actively feeding Arctic char and, with the exception of the spleen, most organs were remarkably unaffected. Whether this reflects a recent dissemination of the parasite within the vascular system or a higher tolerance of char to this parasite than Atlantic salmon remains to be demonstrated. The intriguing absence of gross lesions even in heavily affected fish may be an indication of the latter. The histopathological lesions appear to have greater resemblance to those described by Kent et al. (1992) in farmed Chinook salmon in British Columbia, Canada; in the Chinook salmon, most vessels were packed with the organism, while organ lesions were scarce, as in the present case.

The intracellular stages found together with masses of free trophozoites in the capillaries and sinusoids of the liver, kidney and spleen represent a hitherto undescribed pathomorphological manifestation of spironu- cleosis. The occurrence of this stage appears to be limited to the capillaries and sinusoids of the liver, kidney and spleen, since only characteristic, free-swimming trophozoites were found in the vasculature of other organs. The host cells have not been identified, and both leucocytes and organ-specific cells are possibilities. The appearance of the flagellates as viewed by LM and TEM indicate that they are truly intracellular and not undergoing any decomposition in the host cells. The varying number of parasitic cells within the host cells suggests that they multiply within these cells. A special feature of the intracellular stages was the characteristic 'wrapped-up' appearance. This has previously been observed in a specimen of Spironucleus torosa from the burbot Lota lota (Sterud 1998) (Fig. 19).

Intracellular stages of Octomitus salmonis (syn. Hexamita salmonis) in intestinal endothelial cells were described in the original species descriptions (Moore 1922, Davis 1926). Since then, intracellular hexamitids have not been reported, and the observations made by Moore (1922) and Davis (1926) have been questioned and regarded as misinterpretations (Becker 1977). The present observations of intracellular stages of Spironucleus barkhanus indicate that the observations of similar stages in $O$. salmonis may well have been correct.

The source of infection in the present case of systemic spironucleosis was most probably wild Arctic char. Char smolt used by the affected farm are purchased from a hatchery using water in which wild Arctic char harbour Spironucleus barkhanus (authors' pers. obs.). Although S. barkhanus has never been found in fish within the hatchery, extensive investigations are lacking. Thus, infections with low prevalence may remain undetected. The possibility that only intracellular stages are present for shorter or longer periods may increase the chances of overlooking an infection.

Sterud \& Poynton (2002) anticipated that new cases of spironucleosis were probable and the case pre-

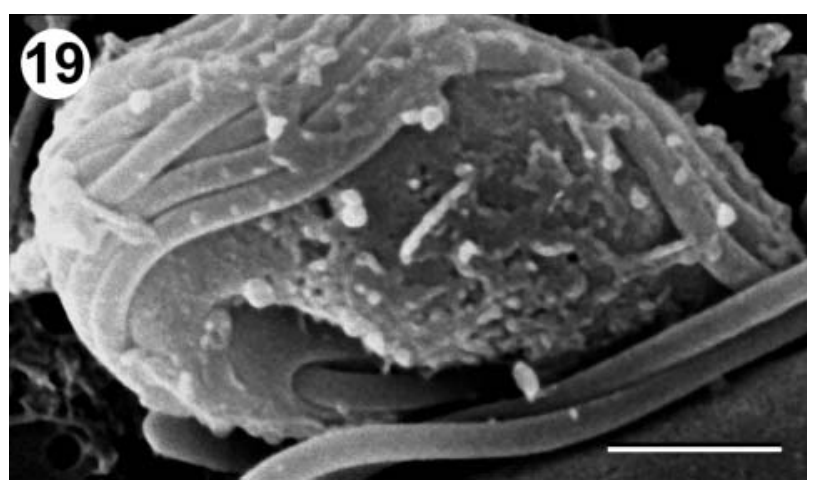

Fig. 19. A 'wrapped-up' individual of Spironucleus torosa from burbot Lota lota (cf. with intracellular stages of $S$. barkhanus in Figs. 15 to 17). Scale bar $=1 \mu \mathrm{m}$. (After Sterud 1998) 
sented in this paper confirms that prediction. With the present findings we have increased our knowledge of the biology of diplomonads infecting fish, but we still believe that further studies of these parasites are needed. This applies especially to the rediscovered intracellular stages.

Acknowledgements. We would like to thank Erna Sommerhaug for giving information and providing biological material, and Dr. Duncan Colquhoun for comments to the manuscript.

\section{LITERATURE CITED}

Becker CD (1977) Flagellate parasites of fish. In: Kreier JP (ed) Parasitic Protozoa, Vol 1. Academic Press, New York, p 404-416

Davis HS (1926) Octomitus salmonis, a parasitic flagellate of trout. Bull Bur Fish Wash 42:9-26

Keister DB (1983) Axenic culture of Giardia lamblia in TYI-S33 medium supplemented with bile. Trans R Soc Trop Med Hyg 77:487-488

Kent ML, Ellis J, Fournie JW, Dawe SC, Bagshaw JW, Whitaker DJ (1992) Systemic hexamitid (Protozoa: Diplo-

Editorial responsibility: Wolfgang Körting,

Hannover, Germany monadida) infection in pen-reared chinook salmon Oncorhynchus tshawytscha. Dis Aquat Org 14:81-89

Mo TA, Poppe TT, Iversen L (1990) Systemic hexamitosis in salt-water reared Atlantic salmon (Salmo salar L.). Bull Eur Assoc Fish Pathol 10:69

Moore E (1922) Octomitus salmonis, a new species of intestinal parasite in trout. Trans Am Fish Soc 52:74-97

Poppe TT, Mo TA, Iversen L (1992) Disseminated hexamitosis in sea-caged Atlantic salmon Salmo salar. Dis Aquat Org 14:91-97

Sterud E (1998) Electron microscopical identification of the flagellate Spironucleus torosa (Hexamitidae) from burbot Lota lota (gadidae) with comments upon its probable introduction to this freshwater host. J Parasitol 84:947-953

Sterud E, Poynton SL (2002) Spironucleus vortens (Diplomonadida) in the ide, Leuciscus idus (L.) (Cyprinidae) a warm water hexamitid flagellate found in Northern Europe. J Eukaryot Microbiol 49:137-145

Sterud E, Mo TA, Poppe TT (1997) Ultrastructure of Spironucleus barkhanus n. sp. (Diplomonadida: Hexamitidae) from grayling Thymallus thymallus (L.) (Salmonidae) and Atlantic salmon Salmo salar L. (Salmonidae). J Eukaryot Microbiol 44:399-407

Sterud E, Mo TA, Poppe TT (1998) Systemic spironucleosis in sea-farmed Atlantic salmon Salmo salar, caused by Spironucleus barkhanus transmitted from feral Arctic char Salvelinus alpinus? Dis Aquat Org 33:63-66

Submitted: January 14, 2003; Accepted: April 1, 2003

Proofs received from author(s): August 21, 2003 